\title{
A PLANAR FACE ON THE UNIT SPHERE OF THE MULTIPLIER SPACE $M_{p}, 1<p<\infty$
}

\author{
CHARLES FEFFERMAN AND HAROLD S. SHAPIRO
}

\begin{abstract}
The unit sphere of the Banach space $M_{p}$ of Fourier multipliers, $1<p<\infty$, is shown to contain a flat portion, i.e. a portion of a plane having codimension one. The proof is based on an elementary inequality, a generalization of the classical Bernoulli inequality.
\end{abstract}

1. Introduction. A complex sequence $\lambda=\left\{\lambda_{n}\right\}_{n=-\infty}^{\infty}$ is a multiplier (on Fourier transforms of elements of $\left.L^{p}(T)\right)$ if its $M_{p}$-norm, defined as

$$
\sup \left(\left(\int\left|\sum \lambda_{n} c_{n} e^{i n t}\right|^{n} d t\right)^{1 / p} /\left(\int\left|\sum c_{n} e^{i n t}\right|^{p} d t\right)^{1 / p}\right)
$$

over all complex sequences $\left\{c_{n}\right\}$ with only finitely many nonzero entries, is finite. Here $1 \leqq p \leqq \infty$, the integration is over the circle $\boldsymbol{T}$, and $d t$ stands for normalized Haar measure. The following facts about the Banach spaces $M_{p}$ are well known (see for instance [1, Chapter 16]).

(a) $M_{p}=M_{p^{\prime}}$ for $p^{\prime}=p /(p-1)$.

(b) For $1 \leqq p<r \leqq 2, M_{p} \subset M_{r}$ (the inclusions being strict).

(c) $M_{1}$ is isometrically isomorphic (via Fourier transformation) to the Banach space $M(T)$ of bounded complex measures on $T$.

(d) $M_{2}=l^{\infty}$ (here $l^{\infty}=L^{\infty}(Z)$ denotes the Banach space of bounded complex sequences) with equality of norms.

Sometimes it will be convenient to refer, rather than to $\lambda \in M_{p}$, to the dual object (distribution) on $\boldsymbol{T}$, conceived as a convolution operator from $L^{p}(\boldsymbol{T})$ to $L^{p}(\boldsymbol{T})$.

2. The object of this note is to prove

THEOREM 1. For $1<p<\infty$, there exists a constant $\alpha(p)>0$ such that whenever $\lambda \in M_{p}$ satisfies $\lambda_{0}=0,\|\lambda\| \leqq \alpha(p)$, the norm of the multiplier

in $M_{p}$ is 1 .

$$
\left(\cdots, \lambda_{-2}, \lambda_{-1}, 1, \lambda_{1}, \lambda_{2}, \cdots\right)
$$

Received by the editors January 24, 1972.

AMS 1970 subject classifications. Primary 42A18; Secondary 26A86.

Key words and phrases. Fourier multiplier, unit sphere, Bernoulli inequality.

(C) American Mathematical Society 1973 
Remarks. (1) Denoting by $\mu$ the sequence $\left\{\mu_{n}\right\}$,

$$
\begin{aligned}
\mu_{n} & =0, & & n \neq 0, \\
& =1, & & n=0
\end{aligned}
$$

(Fourier transform of the Haar measure on $T$ ), it is clear that $\mu$ is a point on the unit sphere $S_{p}$ of $M_{p}$. Consider the hyperplane

$$
H=\left\{\lambda \in M_{p}: \lambda_{0}=0\right\} .
$$

$H+\mu$ is a supporting hyperplane (of complex codimension one) to $S_{p}$ at $\mu$, and the geometric interpretation of Theorem 1 is that $S_{p}$ contains a portion (relatively open nonempty subset) of this hyperplane.

(2) For $p=2$, in view of (d), the theorem is trivial, with $\alpha(p)=1$. In view of (a), therefore, we may (and shall) carry out the proof under the assumption $2<p<\infty$.

(3) The theorem is false for $p=1$ (or $\infty)$; indeed $S_{1}$ does not even contain a (complex!) line segment. ${ }^{1}$

(4) The theorem is valid (with the same proof) for the corresponding real $M_{p}$ spaces; also (with obvious reformulation) when $T$ is replaced by any compact Abelian group.

The proof is based on an extension of Bernoulli's inequality.

Lemma. Let $2<p<\infty$. There exist positive numbers $a_{p}, b_{p}, A_{p}, B_{p}$ such that, for every complex number $z$,

$$
\begin{aligned}
& |1+z|^{p} \geqq 1+p \operatorname{Re} z+a_{p}|z|^{2}+b_{p}|z|^{p}, \\
& |1+z|^{p} \leqq 1+p \operatorname{Re} z+A_{p}|z|^{2}+B_{p}|z|^{p} .
\end{aligned}
$$

Remarks. (1) The exact range of $a_{p}, b_{p}, A_{n}, B_{p}$ for which these inequalities hold has been determined by L. Leindler [2].

(2) Of course (2.1) and (2.2) hold (trivially) also for $p=2$; notationally it is convenient to exclude this case in the proof.

Proof of lemma. Writing $z=x+i y$ with $x, y$ real, we have

$$
|1+z|^{p}=\left(|(1+x)+i y|^{2}\right)^{p / 2}=\left(1+2 x+x^{2}+y^{2}\right)^{p / 2} .
$$

Hence, introducing the abbreviations $c=p / 2>1, s=x^{2}+y^{2}$ the inequalities to be proved take the form

$$
\begin{aligned}
& (1+2 x+s)^{c} \geqq 1+2 c x+a s+b s^{c}, \\
& (1+2 x+s)^{c} \leqq 1+2 c x+A s+B s^{c},
\end{aligned}
$$

1 This is a consequence of equation (2.1.1) on p. 114 of the recent paper by Sten Kaiser, Representations of tensor algebras as quotients of group algebras, Ark. Mat. 10 (1972), 107-141. 
where $a, b, A, B$ are constants which may depend on $c$. In the following we make no attempt to find optimal values for $a, b, A, B$.

Proof of $\left(2.1^{\prime}\right)$. We consider separately the cases $s \geqq 32$ and $s<32$.

Case (i). $s \geqq 32$. It is easy to check that, for $s \geqq 32,2 x+(s / 2) \geqq 0$; hence the left side of $\left(2.1^{\prime}\right)$ is not less than

$$
(1+(s / 2))^{c}>(s / 2)^{c}=(1 / 4)(s / 2)^{c}+(3 / 4)(s / 2)^{c} .
$$

Now, for $s \geqq 32$,

$$
\begin{aligned}
(1 / 4)(s / 2)^{c} & =2^{-c-3} s^{c}+2^{-c-3} s^{c} \\
& \geqq 2^{-c-3} s^{c}+2^{4 c-4}+2^{-c-4} s \geqq C_{1} s^{c}+1+C_{2} s
\end{aligned}
$$

where $C_{1}$ and $C_{2}$ depend only on $c$. Thus $(1 / 4)(s / 2)^{c}$ dominates three of the four terms on the right side of $\left(2.1^{\prime}\right)$. Now in view of $\left({ }^{*}\right)$ we have only to check that $(3 / 4)(s / 2)^{c} \geqq 2 c x$ whereby it is clearly sufficient to restrict attention to $x \geqq 0$. We consider two subcases, $x \leqq 6$ and $x \geqq 6$.

If $x \leqq 6$,

$$
(3 / 4)(s / 2)^{c} \geqq(3 / 4)(16)^{c}>(3 / 4)(16 c)=12 c \geqq 2 c x .
$$

If $x \geqq 6$,

$$
(3 / 4)(s / 2)^{c} \geqq(3 / 4)\left(x^{2} / 2\right)^{c} \geqq(3 / 4) 3^{c} x^{c}>(3 / 4)(3 c) x>2 c x,
$$

completing the proof in Case (i).

Case (ii). $s<32$. By the classical Bernoulli inequality [3, p. 34],

$$
(1+2 x+s)^{c} \geqq 1+c(2 x+s)
$$

and for $s<32$ the right side exceeds $1+2 c x+(c / 2) s+(c / 2)(32)^{1-c} s^{c}$ which is $\left(2.1^{\prime}\right)$ with $a=c / 2, b=(c / 2)(32)^{1-c}$. This completes the proof of $\left(2.1^{\prime}\right)$.

Proof of $\left(2.2^{\prime}\right)$. First let us consider the function

$$
f(t)=\left(\left(1+2 t+t^{2}\right)^{c}-1-2 c t\right) /\left(t^{2}+|t|^{2 c}\right)
$$

for real values of $t$. By Bernoulli's inequality it is nonnegative, and as $t \rightarrow 0$ it tends to the finite limit $2 c^{2}-c$. Also, it tends to 1 as $t \rightarrow \pm \infty$. Hence it is bounded by some positive number $M$ (which depends on $c$ ), and we have

$$
\left(1+2 t+t^{2}\right)^{c} \leqq 1+2 c t+M\left(t^{2}+|t|^{2 c}\right), \quad \text { all } t \in R .
$$

Now it is easy to prove $\left(2.2^{\prime}\right)$. It is convenient to write $s=r^{2}$, where $r \geqq 0$; clearly

$$
-r \leqq x \leqq r .
$$

Observe that $\left(2.2^{\prime}\right)$ is equivalent to the proposition: for each fixed value of $r \geqq 0$, the function

$$
g(x)=1+2 c x+A r^{2}+B r^{2 c}-\left(1+2 x+r^{2}\right)^{c}
$$


is nonnegative for $x$ in the range (2.4). Now, treating $r$ as a constant, we have

$$
g^{\prime \prime}(x)=-4 c(c-1)\left(1+2 x+r^{2}\right)^{c-2}<0 ;
$$

hence the proposition follows once we check that $g(r)$ and $g(-r)$ are nonnegative, that is,

$$
\left(1+2 r+r^{2}\right)^{c} \leqq 1+2 c r+A r^{2}+B r^{2 c}
$$

and the corresponding inequality with $r$ replaced by $-r$. But these follow from.(2.3), with $A=B=M$. This yields (2.2'), and concludes the proof of the lemma.

Proof OF Theorem 1. With the lemma in hand, the rest of the proof is nearly a duplication of one given in [4] for the case $p=4$; for the convenience of the reader, we reproduce the argument here.

An immediate consequence of the lemma is:

If $\rho$ denotes a positive measure of total mass one on some measure space, and $\phi$ a complex-valued function satisfying $\int(\operatorname{Re} \phi) d \rho=0$, then, for $2<p<\infty$,

$$
\begin{aligned}
& \int|1+\phi|^{p} d \rho \geqq 1+a_{p} \int|\phi|^{2} d \rho+b_{p} \int|\phi|^{p} d \rho, \\
& \int|1+\phi|^{p} d \rho \leqq 1+A_{p} \int|\phi|^{2} d \rho+B_{p} \int|\phi|^{p} d \rho .
\end{aligned}
$$

Here $a_{p}, b_{p}, A_{p}, B_{p}$ denote the same constants as in (2.1) and (2.2).

Now, denoting by $u$ the distribution on $T$ defined by $\hat{u}(n)=\lambda_{n}$, Theorem 1 may be reformulated thus: there exists a constant $\alpha(p)>0$ such that if $\hat{u}$ has $M_{p}$-norm not exceeding $\alpha(p)$, and $f$ is any trigonometric polynomial,

$$
\int|(1+u) * f|^{p} d t \leqq \int|f|^{p} d t
$$

(here $d t$ is normalized Haar measure on $\boldsymbol{T}$, and " $l$ " is the function identically equal to 1$)$.

To prove (2.7) we may clearly assume $1 * f=\hat{f}(0) \neq 0$ (hence, by homogeneity, we may assume $\hat{f}(0)=1$ ), since otherwise (2.7) holds whenever $\hat{u}$ has $M_{p}$-norm $\leqq 1$. Thus, write $f=1+g$, where $\hat{g}(0)=0$. The proposition to be proved now reads:

$$
\int|1+(u * g)|^{p} d t \leqq \int|1+g|^{p} d t .
$$

Substituting $u * g$ for $\phi$, and $d t$ for $d \rho$, in (2.6) gives

$$
\int|1+(u * g)|^{p} d t \leqq 1+A_{p} \int|u * g|^{2} d t+B_{p} \int|u * g|^{p} d t .
$$


Now, assuming that convolution with $u$ is a bounded operator from $L^{p}$ to $L^{p}$ with norm $\leqq \alpha$, and recalling that the $L^{2} \rightarrow L^{2}$ norm of a convolution operator cannot exceed the $L^{p} \rightarrow L^{p}$ norm, we get from (2.9)

$$
\int|1+(u * g)|^{n} d t \leqq 1+A_{p} \alpha^{2} \int|g|^{2} d t+B_{p} \alpha^{p} \int|g|^{p} d t
$$

But, from (2.5), with $\phi=g, d \rho=d t$,

$$
\int|1+g|^{\prime \prime} d t \geqq 1+a_{p} \int|g|^{2} d t+b_{p} \int|g|^{p} d t .
$$

Comparing (2.10) and (2.11), we see that the desired inequality (2.8) holds if $A_{p} \alpha^{2} \leqq a_{p}, B_{p} \alpha^{p} \leqq b_{p}$. Thus (2.8) holds if the $M_{p}$-norm of $\hat{u}$ does not exceed $\alpha .(p)$, where

$$
\alpha(p)=\min \left[\left(a_{p} / A_{p}\right)^{1 / 2},\left(b_{p} / B_{p}\right)^{1 / p}\right],
$$

completing the proof of Theorem 1.

A final remark: We have exhibited a planar-face on the unit sphere $S_{p}$ of $M_{p}$ passing through one particular point $\mu$ (the sequence corresponding to Haar measure on $\boldsymbol{T}$ ); of course, then there is also a face containing $z \mu$, where $z$ is any complex number of modulus one. But, are there other essentially different ones? It might be of some interest to investigate this, and the related question: which points of $S_{n}$ lie in the (relative) interior of planar faces?

\section{BIBLIOGRAPHY}

1. R. E. Edwards, Fourier series: A modern introduction. Vol. II, Holt, Rinehart and Winston, New York, 1967. MR 36 \#5588.

2. L. Leindler, On a generalization of Bernoulli's inequality, Acta Sci. Math. (Szeged) (to appear).

3. D. S. Mitrinović, Analytic inequalities, Die Grundlehren der math. Wissenschaften, Band 165, Springer-Verlag, Berlin and New York, 1970. MR 43 \#448.

4. H. S. Shapiro, Fourier multipliers whose multiplier norm is an attained value, Proc. Conference Linear Operators and Approximation (Oberwolfach, Germany, 1971) (to appear).

Department of Mathematics, University of Chicago, Chicago, Illinois 60637 (Current address of Charles Fefferman)

Department of Mathematics, University of Michigan, AnN Arbor, Michigan 48104

Current address (Harold S. Shapiro): Mathematics Institute, Royal Institute of Technology, 10044 Stockholm 70, Sweden 